\title{
Smart Efficient Flame Retardant Carpets in non Halogen Flame Retardant Polymers
}

\author{
Xiaodan Lin *, Bin Yang, Guangkai Wang, Erigene Bakangura, Ming Huang, \\ Zhishan Fan
}

\author{
College of Materials Sci. and Eng., South China Univ. of Tech., Guangzhou 510640, PR China \\ *mcxdlin@scut.edu.cn
}

Keywords: Flame retardant; Wetting; Self-assemble; Red phosphorus; Oxygen shielding screen

\begin{abstract}
Non halogen flame retardant researches have achieved great progress; however, theoretical works are much lagged. Here we show a model of an efficient Flame Retardant Carpet (e-FRC) based on the synergistic flame retardant system of phosphorus and hydroxyl compounds that can give ABS UL 94 V-0 efficiently. This model is further extended to explain the high efficient flame retardancy of phosphonates by proposing a surfactant structured intermediate from pyrolysis. The smart intermediate self-assembles on charred polymer matrix, forming an anisotropy molecular membrane, with one side of hydrocarbon groups and the other of inorganic groups. The organic side adheres onto polymeric char, and inorganic side facing the fire, forms an Oxygen Shielding Screen (OSS). The OSS together with charred layer underneath constitutes an e-FRC. This e-FRC structure can be found in earlier reports. It can explain all present existing efficient flame retardant systems.
\end{abstract}

\section{Introduction}

Contamination by the use of halogen flame retardants onto the ecosphere is widespread (1), and brominated flame retardant is even ubiquitous in arctic area (2). There is an urgent need for substitutions. Because of the diversity of flame retarded polymers and the complexity of the combustion process, and also an efficiency FR is never achieved by one single mechanism, a number of the flame retardant researches are purely empirical. Comparing with the achievements of practical researches, theoretical works on non halogen flame retardancy are much lagged.

In 1988, Iji and Serizawa (3) found a phenyl-rich silicone mixture is effective in retarding the combustion of PC, and proposed that the superior flame retardancy is belong to its excellent dispersion in PC and the rapid movement to the surface of PC to form the highly flame resistant char barrier during combustion. Other efficient flame retardant additive for PC have been reported much earlier in 1977 in a USP (4), 1 part of alkali or alkaline earth metal salt of an inorganic sulfur compound can give PC V-II grade; more efficient perfluorobutane sulfonate was patented in US in 1995 (5), although fluoro compound, its effect addition is only 0.01-0.1\%. Later researchers (6) ascribe its majority flame retardant activity to condensed phase activity. However, no further study was seen as how does it acting in the condensed phase. In 1996, Harrocks (7) find that well coated intumescent charred fibers can withstand temperature up to $1200{ }^{\circ} \mathrm{C}$ for 5 minutes. They proposed a "char-bond" model and related it to the good compatibility of intumescent char with the fibers, which enable it to prevent the infiltration of oxygen.

In 2008, for the highly flammable ABS, traditional halogen flame retardant fortress, Hoang et al (8), prepared a series cyclo phosphonates and phosphates and found that for all phosphonates, $15 \%$ addition can give ABS UL $94 \mathrm{~V}-0$ performance. However, whether the governing mechanism is gas phase or condensed phase or both is not clear. Actually, some volatile phosphorated compounds are very efficient combustion inhibitors. They are even a few times more efficiency than bromine compounds (9). Recently, Brehme and Schartel et al. (10) studied the fire behavior of a phosphorus containing polyester (PET-P-DOPO). They quantitatively identified the contribution of three different mechanisms: flame inhibition, charring and a protection effect by the intumescent char. Their study shows a big portion of flame inhibition contribution in view of cone calorimeter data.

Apart from the controversial issue on gas phase or condensed phase action, we may ask whether there is a resembling chief governing flame retardant mechanism in those efficient FR systems, what is it? 


\section{The "Hands" that Spread and Weave an efficient Flame Retardant Carpet in ABS}

In 2011, Wang and Lin et al. (11) found that micro encapsulated red phosphorus (MRP) with 85\% phosphorus encapsulated by melamine resin and benzoxazine (BOZ) system shows good synergistic effect. It is obvious, MRP alone cannot give UL $94 \mathrm{~V}-0$ performance up to $20 \%$ loading, however, when it is combined with BOZ, V-0 can be achieved at $20 \%$ loading with $\mathrm{MRP} / \mathrm{BOZ}$ between $2 / 3 \sim 3 / 2$, and also with $15 \%$ of MRP and $\mathrm{BOZ}$ plus $0.3 \% \mathrm{Al}(\mathrm{OH})_{3}$; and down to $12 \%$ with ready hydroxyl rich novolac (12) at MRP/novolac of 2/3 3/2.

Benzoxazine resins are high char residue resin (13) and phosphorus containing benzoxazine show high flame retardant property $(14,15)$. The high synergistic effect suggests that BOZ is not a simple char former. The hydroxyl group has played an important role in synergistic flame retardancy of ABS.

SEM pictures (Fig. 1A-E) of char residues left by UL 94 V-0 samples show two typical textures: solid skin layer and perfect foam; and the two textures could be perfectly combined as shown in fig. 1E. Fig. 1A is burnt region after UL 94 test of MRP/BOZ/ABS composite. It shows a thin skin carpet formed during burning of the sample. From the edges of fractured carpet, we could measure that the carpet thickness is in micrometers (around $2 \mu \mathrm{m}$ ). This picture also shows that there are secondary carpets underneath. The bent curved char carpet and the thickness resemble the char structure reported by Brehme and Schartel et al. (10). It seems that if the top carpet is destroyed, a new carpet underneath is formed instantly, thus complete a well flame retardant. This solid skin carpet can be seen in the char from MRP/novolac/ABS composite too (Fig. 1B). Besides the solid char skin texture, the more often seen char structure is foamed char in Fig. 1C and Fig. 1D found in $\mathrm{BOZ}$ and novolac system respectively. Fig. 1A and Fig. 1C are of the same composition, but totally different structure. It must be formed during different combustion stages, or variation in compositions, etc. The complexity of char morphology remains a scientific problem. Other report has shown the fine foam char texture before and it was "sponge structure" in the report of Price et. al(16). The solid skin structure and foam structure combined gradually and smoothly in Fig. 1E. Both solid skin layer and foam structure together with their different combinations contributed to the valid flame retardancy of the composites.
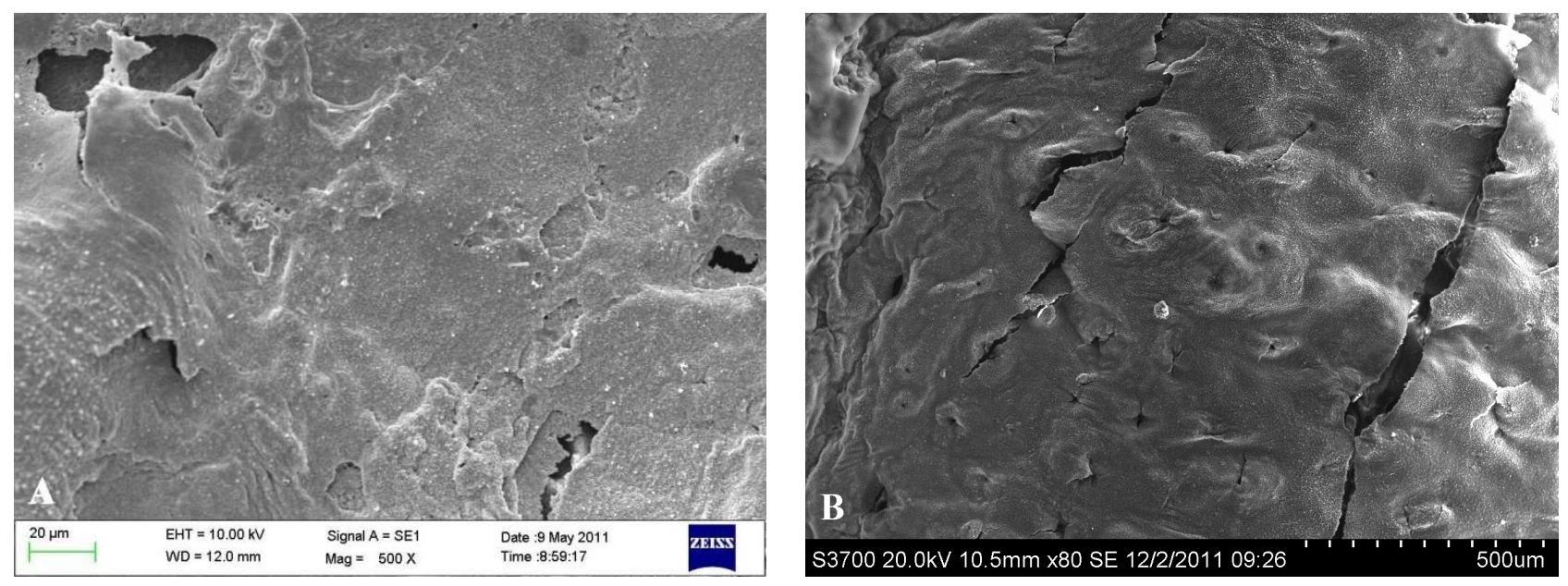

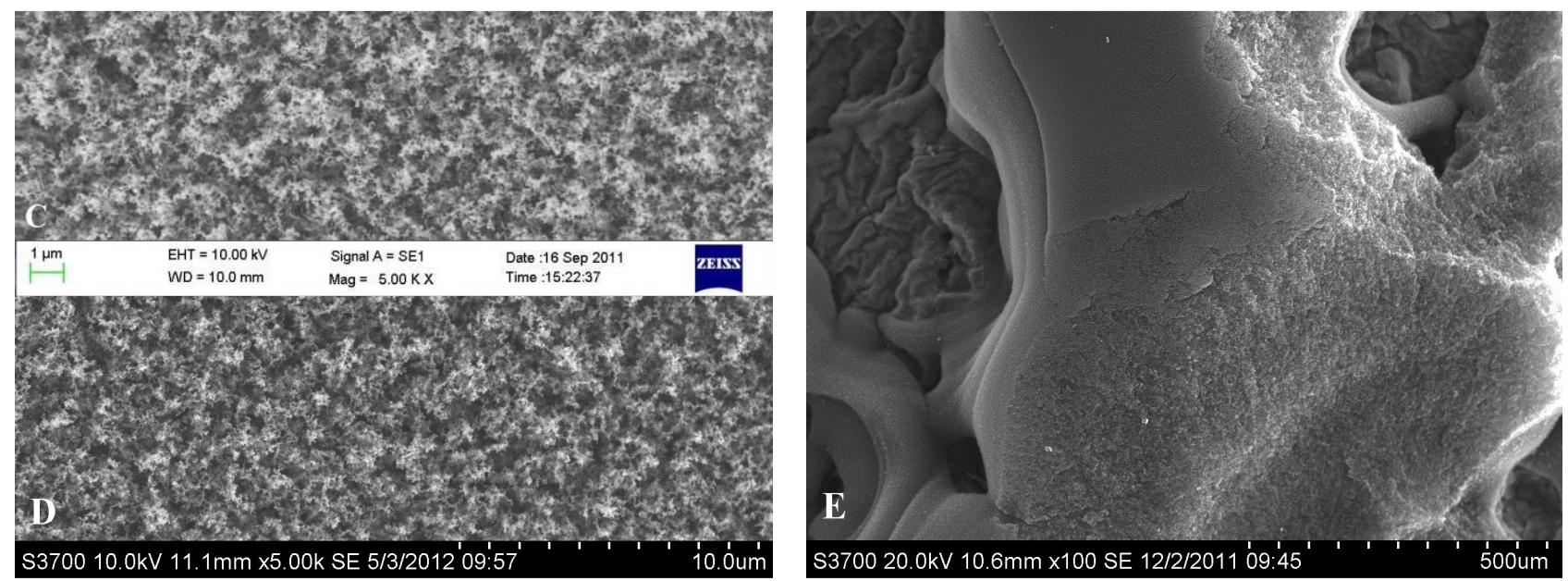

Figure 1. SEM pictures of char residues left by UL $94 \mathrm{~V}-0$ samples. A: Burnt region of

MRP/BOZ/ABS (8/12/80); B: MRP/novolac/ABS (6.0/9.0/85), C: MRP/BOZ/ABS (8/12/80); D: MRP/novolac/ABS (9/6/85), E: MRP /novolac/ABS (6.0/6.0/88)

Although the relationship between char structure, char composition, and composite composition and flame behavior are far from clear, these pictures inspirited us to propose the synergistic mechanism below in scheme 1 .
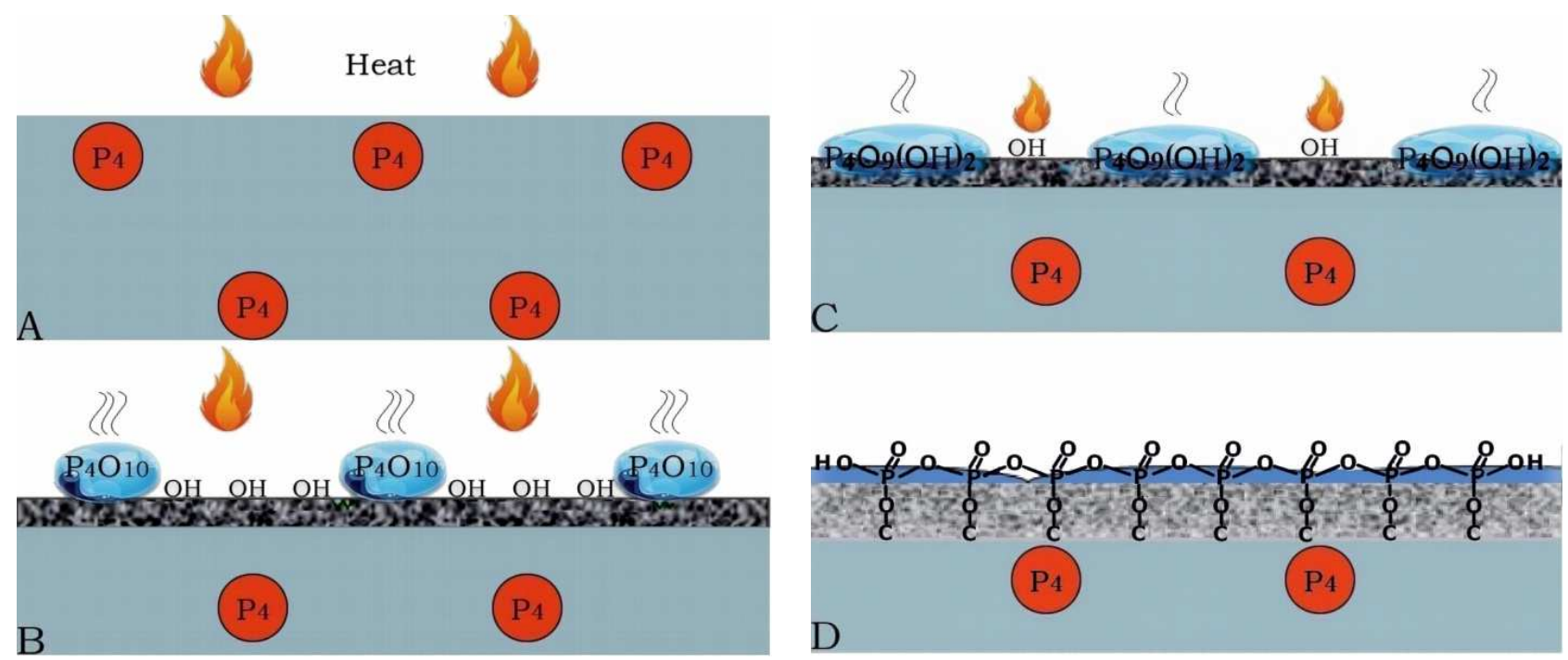

Scheme 1. The formation of an e-FRC in MRP/BOZ/ABS. A: Start state of MRP (in red circle, $\mathrm{P}_{4}$ ) filled BOZ/ABS composite; $\mathrm{B}$ : MRP is oxidized to phosphorus pentoxide (blue ellipse, $\mathrm{P}_{4} \mathrm{O}_{10}$ ) and $\mathrm{BOZ}$ ring opened and form a lot of hydroxyl which dispersed in the charred BOZ/ABS; $\mathrm{C}$ :

Phosphoric acid wetting the hydroxyl char; D: Phosphoric acid condensed and esterifies with hydroxyl char forming an OSS, which together with the char form an e-FRC.

Scheme 1A represent the original state of MRP filled BOZ/ABS composite. While being heated by fire, MRP oxide to phosphorus pentoxide, and BOZ ring opened and polymerized form hydroxyl rich polymer, which further oxide to a hydroxyl containing char (scheme 1B). It is believed that the uniform distributed hydroxyl "char", which is come from good compatibility of BOZ with ABS, is good to synergistic with $\mathrm{P}_{2} \mathrm{O}_{5}$ : to liquidates the highly viscous $\mathrm{P}_{2} \mathrm{O}_{5}$, reduce its sublimation and evaporation as seen in scheme $1 \mathrm{C}$, enable it could wet the hydroxyl charred composite surface, and be anchored there tightly by esterifies with the hydroxyl char materials and condensed at the same time, forming an e-FRC as shown in scheme 1D.

The formation of P-O-C has been well proved by a number of researchers. A close report was given by Hatsuhiko et al. (17). The anchored phosphoric compounds condensed on the charred surface, forming an OSS, which shields away the attacking hot oxygen and hydroxyl radicals. Small 
quantity of aluminum hydroxide makes the anchoring even more strong, so long as it not petrifies the wetting ability of phosphoric acids. By this way, an e-FRC formed. Here, the "hands": wetting, charring, esterifying and metal ion anchoring have spread and woven an e-FRC.

Is this OSS armed char e-FRC exists in other systems?

\section{The high efficiency of phosphonates-smart surfactant structure intermediate and formation of OSS and e-FRC}

In 2008, Hoang et al (8) found that for all phosphonates, 15\% addition can give ABS to reach UL $94 \mathrm{~V}-0$. However, phosphates failed, and PDPDP (Pentaerythritol diphenyl diphosphate) failed even with $35 \mathrm{wt} \%$ loading. The advantages of phosphonates superior to that of phosphates in char residue can also be found in the report data of Price et al. (16) and Adina and Bob (18); and phosphonates are known as very efficient flame retardants $(10)$.

To explain the more efficiency of phosphonates than phosphates, we proposed a surfactant structure intermediate that can self assemble an OSS, which is shown in scheme 2.
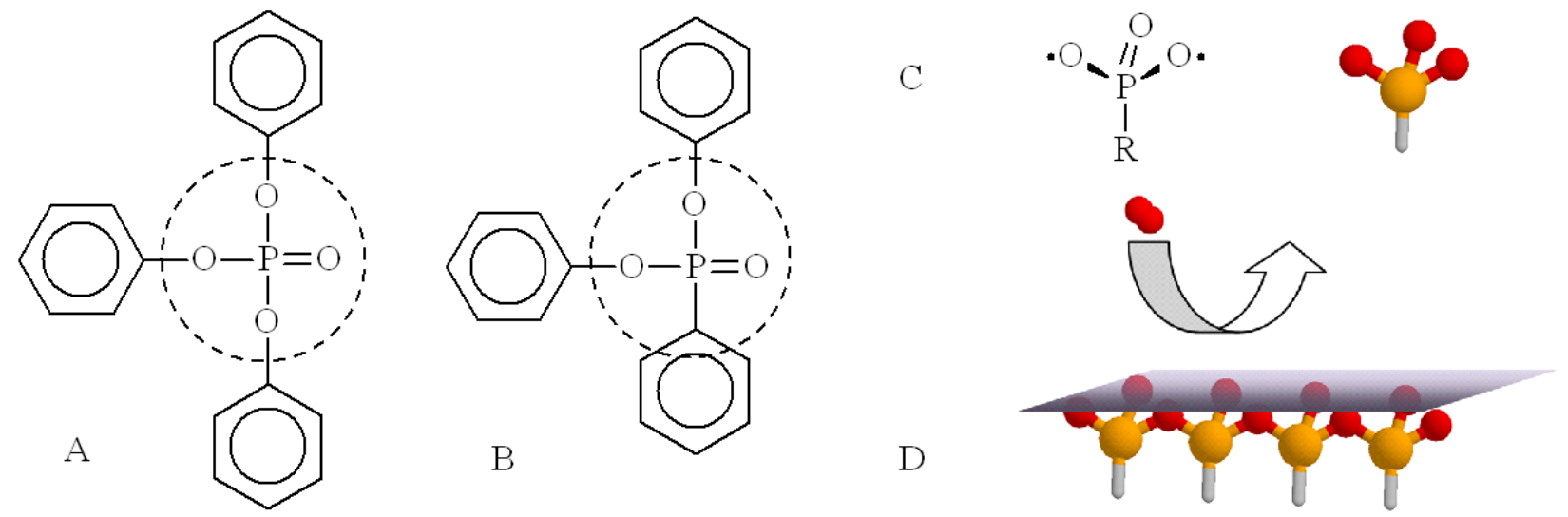

Scheme 2. The formation of an OSS. A: a simple phosphate; B: a simple phosphonates; C: possible smart surfactant structure from the pyrolysis of B (R represents hydrocarbon radicals, red ball represents oxygen atom, yellow ball represents phosphorus atom); D: smart surfactant structure intermediate $\mathrm{C}$ and its self-assembled OSS.

In scheme $2 \mathrm{~A}$ and $\mathrm{B}$, although $\mathrm{C}-\mathrm{O}$ bond energy is higher than $\mathrm{C}-\mathrm{P}$, considering the random behavior of pyrolysis, the smart surfactant structure intermediate in scheme $2 \mathrm{C}$ will resulted in reasonable quantity. Further considering the chemical environments and phosphorus centered stereo effect (see the dot line circle), the closely connected phosphonates (scheme 2 B) get more chance to be preserved in certain temperature, forming the smart intermediate with structure like surface active agent as shown in scheme $2 \mathrm{C}$. The pyrolysis of phosphates (A) get more chance to pyrolysis to inorganic phosphoric acid which tend to aggregate on the surfaces of organic polymers and most polymer chars. The aggregation of phosphoric compounds greatly decreased its flame retardancy ability. The smart surfactant like phosphonates can easily spread over the polymer substrate and polymeric char and self-assembled over polymeric char, forming an OSS that protect the polymeric char (which oxidize rapidly above $400{ }^{\circ} \mathrm{C}$ in air (7)) from oxygen attacks (shown in scheme 2D). Thus an e-FRC formed. 


\section{Other existing e-FRC}

In fire fighting, blanket is a very efficient tool. In polymers flame retardancy, the above smart e-FRC has already existed for a long time. Scheme 3 shows three oxygen shielding screen model for typical efficient flame retardant systems.

Scheme 3A represents possible pyrolysis intermediate from a commercialized product aluminum phosphonates. Here the introducing of aluminum atom increased the covering area of oxygen shielding screen, however, it also limited the solubility and mobility of aluminum phosphonates.

In the surfactant structure of scheme $2 \mathrm{C}$, when the center $\mathrm{P}$ is substitute by $\mathrm{Si}$, it forms the core flame retardancy ability of methyl phenyl-silicone (represent in scheme 3B), which is much efficient in PC flame retardancy $(3,5)$; Iji and Serizawa (3) ascribe it to the formation of highly flame resistant "char barrier" during combustion. Now, it is believed that the high efficiency is due to the "char barrier" that is armed with well arranged and structured oxygen shielding screen. When the center $\mathrm{P}$ is substitute by $\mathrm{S}$, the typical example is potassium perfluorobutane sulfonate as shown in scheme $3 \mathrm{C}$. $\quad 0.01 \% \sim 0.1 \%$ addition of potassium perfluorobutane sulfonate in PC $(5,6)$ can give UL 94 V-0 performance. This is a perfect purely surfactant model.

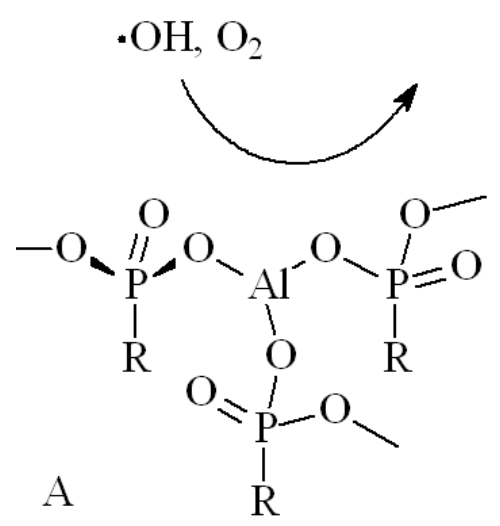

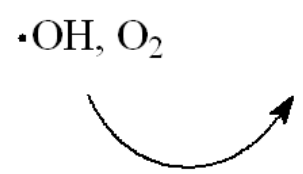

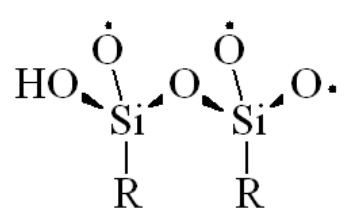

$\mathrm{B}$

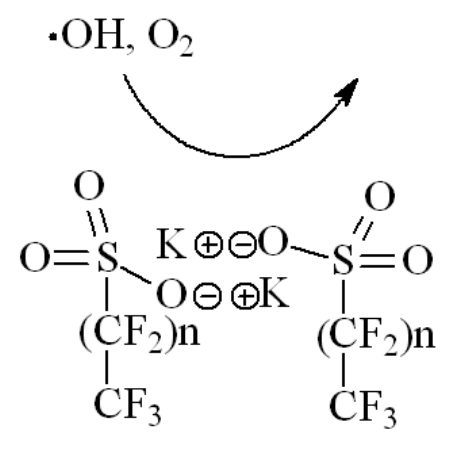

$\mathrm{C}$

Scheme 3. OSS in typical e-FRC systems. All the atoms on the out molecular OSS are oxygen and fully oxidized atoms condensed together.

\section{Summary}

An efficient Flame Retardant Carpet (e-FRC) is composited of a char layer or high thermal stable polymers which are covered tightly in molecular level by seamless Oxygen Shielding Screen (OSS). Wetting and chemical bonding of the oxidized phosphoric compound to hydroxyl function polymeric char help the formation of OSS. Phosphonates and some other organic and inorganic hybrid compounds may pyrolysis to a surfactant structured intermediate that could self-assemble on polymeric char forming an OSS. OSS combines together with polymeric char layer or highly thermal stable polymers forming an e-FRC. So long a seamless e-FRC is formed and stable existed, high flame retardant performance will be resulted.

\section{Acknowledgements:}

The corresponding author thanks Dr. W. L. Cheung of The University of Hong Kong, for his earnest guidance. Thanks also given to the Education Minister of China for a research fund on FR research (2002) and the Sci. and Tech. Bureau of Guangdong Province and Guangzhou City. 


\section{References}

[1] Santillo, D. \& Johnston, P. Playing with fire: the global threat presented by brominated flame retardants justifies urgent substitution. Environment International 29, 725-734 (2003).

[2] de Wit, C. A. Herzke, D. Vorkamp, K. Brominated flame retardants in the Arctic environment trends and new candidates, Science of the Total Environment 408, 2885-2918(2010).

[3] Iji, M. \& Serizawa, S. Silicone derivatives as new flame retardants for aromatic thermoplastics used in electronic devices. Polym. Adv. Technol. 9, 593-600(1998).

[4] Webb, J. L. Novel flame retardant polycarbonate compositions. USP 4,028,297; (1977).

[5] Umeda, T. Nodera, A. Hashimoto, K. Flame retardative polycarbonate resin composition. USP 5,449,710; (1995).

[6] Huang, X. Ouyang, X. Ning, F. Wang, J. Mechanistic study on flame retardance of polycarbonate with a small amount of potassium perfluorobutane sulfonate by TGA-FTIR/XPS. Polymer Degradation and Stability 91, 606-613(2006).

[7] Horrocks, A. R. Anand, S. C. Sanderson, D. Complex char formation in flame retarded fibre-intumescent combinations: 1. Scanning electron microscopic studies. Polymer 37 (15), 3197-3206(1996).

[8] Hoang, D. Q. Kim, J. Jang, B. N. Synthesis and performance of cyclic phosphorus-containing flame retardants. Polymer Degradation and Stability 93, 2042-2047(2008).

[9] Babushok, V. \& Tsang, W. Inhibitor Rankings for Alkane Combustion. Combustion and Flame $123,488-506$ (2000).

[10]Brehme, S. et al. Phosphorus polyester versus aluminium phosphinate in poly(butyleneterephthalate) (PBT): Flame retardancy performance and mechanisms. Polymer Degradation and Stability 96, 875-884(2011).

[11]Wang, G.K. Lin, X.D. Yang, B. Fan, Z.S. Non halogen flame retardant agent containing benzoxazine and their non halogen flame retardant polymer composites. PCT/CN2011/078955.

[12]Yang, B. Lin, X.D. Wang, G.K. Liu, D. D. Xue, L.Z. Non halogen flame retardant agent containing novolac phenol resin and their non halogen flame retardant polymer composites. $\mathrm{CN}$ patent 201110387792.9. Nov. 2011.

[13]Hemvichian, K. \& Ishida, H. Thermal decomposition processes in aromatic amine-based polybenzoxazines investigated by TGA and GC-MS. Polymer 43, 4391-4402(2002).

[14]Espinosa, M.A. Galià, M. Cádiz, V. Novel phosphorilated flame retardant thermosets: epoxy-benzoxazine-novolac systems. Polymer 45, 6103-6109 (2004).

[15] Spontón, M. Lligadas, G. Ronda, J. C. Galià, M. Cádiz, V. Development of a DOPO-containing benzoxazine and its high-performance flame retardant copolybenzoxazines. Polymer Degradation and Stability 94, 1693-1699(2009).

[16]Price, D. et al. Thermal behaviour of covalently bonded phosphate and phosphonate flame retardant polystyrene systems. Polymer Degradation and Stability 92, 1101-1114(2007).

[17]Hatsuhiko, H. Yoshihisa, T. Takahito, I. Synergistic effect of red phosphorus, novolac and melamine ternary combination on flame retardant flame retardancy of poly(oxymethylene). Polymer Degradation and Stability 91, 1996-2002(2006).

[18]Dumitrascu, A. \& Howell, B. A. Flame-retarding vinyl polymers using phosphorusfunctionalized styrene monomers. Polymer Degradation and Stability 96, 342-349(2011). 